\title{
Numerical simulation of full-aperture-pair ion optics in a miniature ion thruster
}

M. Nakano, K. Nakamura, Y. Nakagawa, D. Tomita, Y. Takao, and H. Koizumi

Citation: Physics of Plasmas 25, 013524 (2018);

View online: https://doi.org/10.1063/1.5008802

View Table of Contents: http://aip.scitation.org/toc/php/25/1

Published by the American Institute of Physics

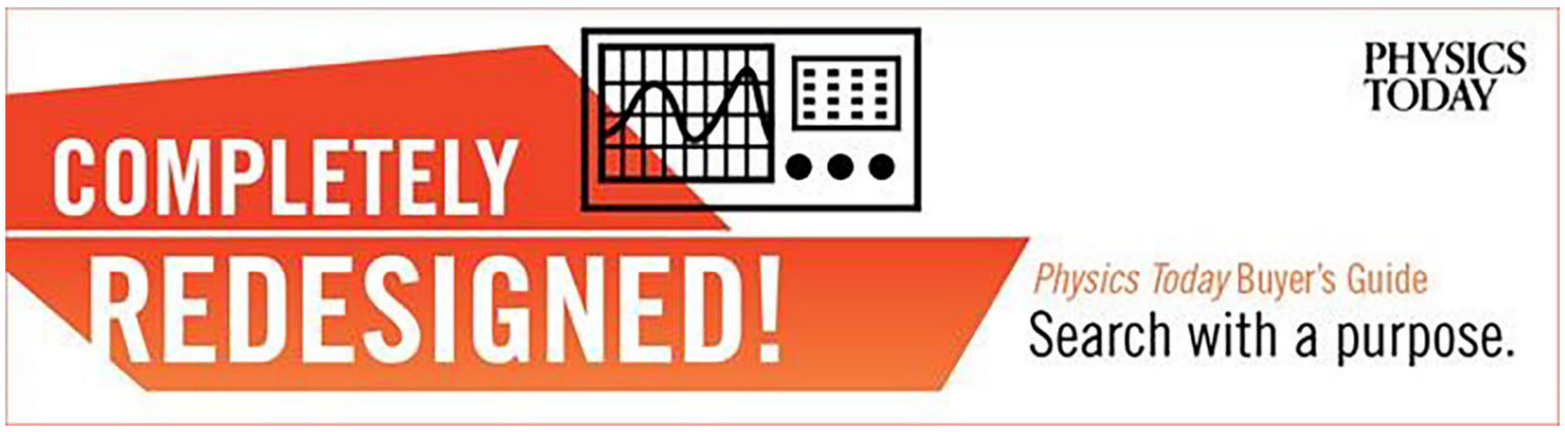




\title{
Numerical simulation of full-aperture-pair ion optics in a miniature ion thruster
}

\author{
M. Nakano, ${ }^{1}$ K. Nakamura, ${ }^{2}$ Y. Nakagawa, ${ }^{3}$ D. Tomita, ${ }^{3}$ Y. Takao, ${ }^{2}$ and H. Koizumi ${ }^{4}$ \\ ${ }^{1}$ Department of Engineering, Tokyo Metropolitan College of Industrial Technology, 8-17-1, Minami-senjyu, \\ Arakawa-ku, Tokyo 113-0852, Japan \\ ${ }^{2}$ Department of Systems Integration, Yokohama National University, 79-5 Tokiwadai, Hodogaya, \\ Yokohama 240-8501, Japan \\ ${ }^{3}$ Department of Aeronautics and Astronautics, The University of Tokyo, 7-3-1 Hongo, Bunkyo-ku, \\ Tokyo 113-8656, Japan \\ ${ }^{4}$ Department of Advanced Energy, The University of Tokyo, 5-1-5, Kashiwanoha, Kashiwa 277-8561, Japan
}

(Received 10 October 2017; accepted 5 January 2018; published online 22 January 2018)

The 211-aperture-pair two-grid ion optics of a miniature ion thruster is numerically simulated. Since the plasma in the miniature ion thruster is too inhomogeneous to introduce mirror or translational boundary conditions between apertures, all the apertures of the grid system are considered. The simulation is self-consistent, the ion current profile in the discharge chamber plasma is given by the particle-in-cell with Monte Carlo collision algorithm calculations, and all the ion beams extracted from the full-aperture-pair array were tracked including charge-exchange ions. A scheme for the construction of the full-aperture-pair simulation domain is proposed based on the array of a six-fold hexagonal single-aperture-pair simulation domain, which can be extended to other numbers of aperture pairs. Numerical results on accel impingement current and ion-beam profile are compared to experimental data and shown to be in reasonable agreement. Furthermore, the full-aperture-pair ion-optics model is compared with the single-aperture-pair ion-optics models used in the majority of previous ion-optics simulations, which showed that the full-aperture-pair ion-optics model yielded the most accurate predictions. These results suggest that the ion thruster grid system using an inhomogeneous plasma source can be designed more accurately and effectively using full-aperture-pair ion-optics simulations. Published by AIP Publishing. https://doi.org/10.1063/1.5008802

\section{NOMENCLATURE}

$A_{\text {down }}$ downstream boundary area

$A_{u p} \quad$ upstream boundary area

$d_{a}, d_{s} \quad$ accel and screen grid diameters

$e$ elementary charge

$j \quad$ ion current density at the upstream surface

$J_{\mathrm{a}}$ accel impingement current: $J_{\mathrm{a}}=J_{\mathrm{ai}}+J_{\mathrm{aCE}}$ $+J_{\mathrm{aEL}}$

$J_{\mathrm{a}}\left(N_{\mathrm{xy}}, N_{\mathrm{z}}\right)$ accel impingement current for $\left(N_{\mathrm{xy}}, N_{\mathrm{z}}\right)$ mesh divisions

$J_{\mathrm{aCE}}$ accel impingement current due to chargeexchange ions

$J_{\mathrm{aEL}}$ accel impingement current due to elasticcollision ions

$J_{\text {ai }}$ accel impingement current due to direct impingement

$J_{\mathrm{b}} \quad$ beamlet current, defined as the current passing through the ion-acceleration grid system

$J_{\text {scl }}$ space charge limited current: $J_{s c l}$ $=\frac{4 \varepsilon}{9} \sqrt{\frac{2 e}{m_{\mathrm{Xe}}}} \frac{\left(\phi_{s}-\phi_{a}\right)^{3 / 2}}{l_{e}^{2}}\left(\frac{\pi d_{s}^{2}}{4}\right)$

$J_{\text {up }}$ ion current that flows into the upstream boundary

$k_{\mathrm{B}}$ Boltzmann constant

$L$ distance to probe

$l_{\text {down }}$ distance to downstream boundary

$l_{e}$ effective acceleration length: $l_{e}=\sqrt{l_{g}^{2}+d_{s}^{2} / 4}$

$l_{g}$ distance between screen and accel grids

$l_{n}$ distance to the potential hill

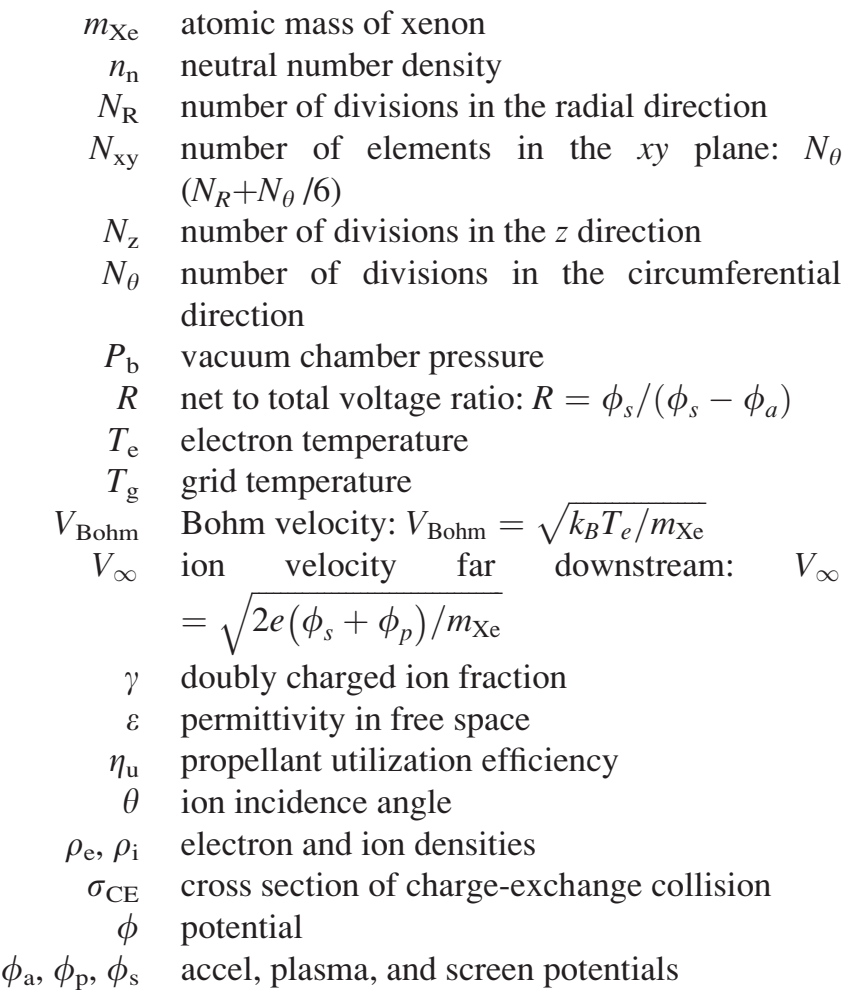

\section{INTRODUCTION}

An ion thruster is a form of electric propulsion running on electrical power. It creates thrust by accelerating ions and 


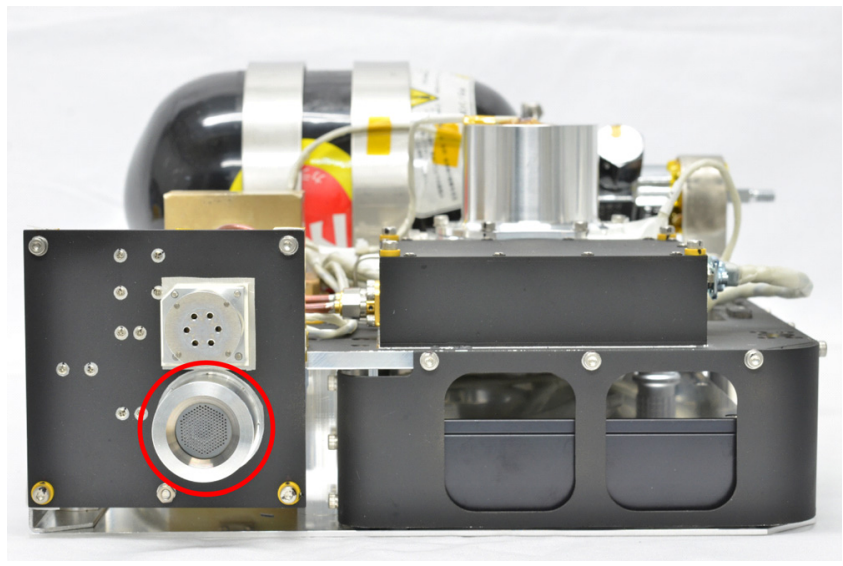

FIG. 1. Front view of a miniature ion propulsion system (MIPS) and a 211aperture-pair grid array (red circle).

offers substantial propellant savings owing to its high specific impulse. Ion thrusters have been used in a variety of space missions such as attitude-altitude controls, GEO satellite insertion, and interplanetary flights. ${ }^{1-5}$

One of the current trends in space propulsion is miniaturization for small spacecraft applications. Ion thrusters are not exceptions in this trend, and they were used in microspacecraft missions such as Hodoyoshi-4 and PROCYON, in which the miniature ion propulsion system (MIPS) shown in Fig. 1 was successfully operated onboard. ${ }^{4,5}$

Owing to the tight schedule of rideshare requirements of microspacecraft, a shorter design, customization, and qualification of ion thrusters including their ion optics are becoming increasingly important. During the design process, numerical simulation helps to guide an experimental study that would otherwise be time consuming and costly because ion optics involves many parameters, such as grid thickness, aperture diameter, and gap.

MIPS is an ion thruster system that employs electron cyclotron resonance (ECR) discharges with ring-shaped permanent magnets and a $4.2-\mathrm{GHz}$ microwave antenna. Figure 2(a) shows a photograph of an MIPS grid array during operation taken from the downstream side of its grid set. The image shows a ring-shaped plasma with a darker region in the center and the periphery of the aperture array. The ECR heating process inside the MIPS discharge chamber was extensively studied by Takeo et al., and it was found that effective heating in the ECR layer downstream of the ringshaped antenna yields a discharge plasma with a similar inhomogeneous ring-shaped profile [Fig. 2(b)]. ${ }^{6}$ This inhomogeneity of the MIPS plasma source is a result of the tradeoff between the optimization of plasma generation and miniaturization for microspacecraft application, which required a significant amount of experimental and numerical effort. Owing to the inhomogeneity of the MIPS plasma shape, the extracted ion beam current and ion incidence angles differ among apertures. Therefore, the approach to optimize single-aperture ion optics is not appropriate; rather, a global approach to analyze full-aperture-pair ion optics is necessary for the optimization of the MIPS ion optics.

There are many papers on ion-optics simulations. The majority of ion-optics models are designed for single-aperture-pair ion optics considering the six-fold hexagonal symmetry of the aperture array. ${ }^{7-12}$ Wang et al. developed a three-dimensional ion-optics model designed in such a way that not only a single aperture but also multiple apertures can be included in the simulation domain; however, their simulations were performed only with two quarter-size apertures. ${ }^{13}$ The model was extended to a 7-aperture grid by several authors, ${ }^{14,15}$ who used the symmetry of the plasma shape and the aperture arrangement to reduce the size of the simulation domain.

Although the single-aperture-pair ion-optics model and the above-mentioned symmetric models yielded reasonable results in most ion-optics calculations, they cannot be applied to a highly inhomogeneous plasma source, since it is difficult to draw a symmetric bound in the grid set. For a simplified case, the effect of inhomogeneity of the plasma source was investigated by Shagayda et al. using 5 half-size and 2 quarter-size apertures, but they assumed the ion current gradient and ion incidence angles as constant.

Another limitation of the single-aperture-pair ion-optics model is that it cannot be applied to peripheral apertures, because its beamlet profile and erosion pattern are not sixfold hexagonal in shape. One example is observed in a 19 aperture-pair ion-optics experiment, in which beamlet profiles at the peripheral apertures have two or three (not six)

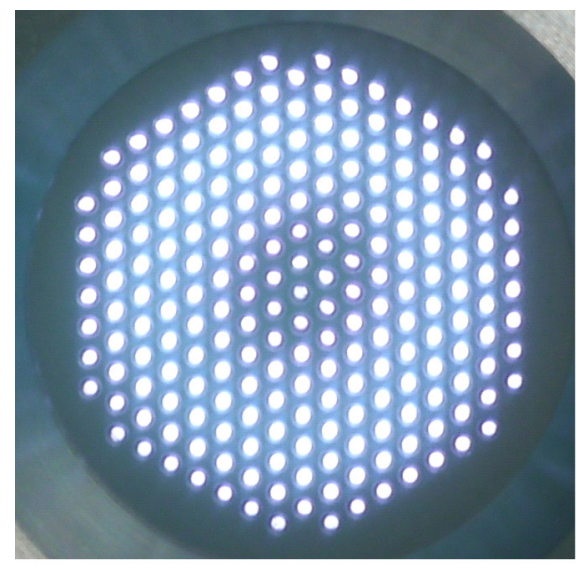

(a)

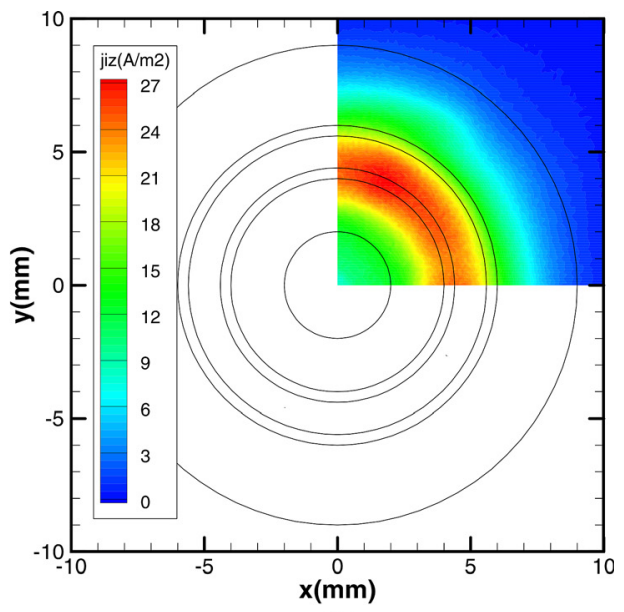

(b)
FIG. 2. (a) Front view of the MIPS grid array during operation. (b) Ion current density distribution in the discharge chamber obtained by PIC-MCC calculations. 
sharp points. ${ }^{16}$ In the MIPS ion optics, because the number of peripheral apertures is 48 (accounting for $23 \%$ of all the apertures), the single-aperture-pair ion-optics model may not be appropriate for evaluation.

In this study, a full-aperture-pair (211-aperture-pair) two-grid ion-optics assembly with a highly inhomogeneous MIPS plasma source was analyzed. The ion current density distribution and the incidence angle of ions obtained by particle-in-cell with Monte Carlo collision algorithm (PICMCC) calculations were used as the inputs of ion-optics simulations. Furthermore, a simple and effective scheme for the construction of a full-aperture-pair simulation domain was proposed, which is based on the array of the single-aperturepair simulation domain.

The objectives of this study are (1) to enable the numerical investigation of full-aperture-pair ion optics for an inhomogeneous plasma source and (2) to conduct comparative evaluations of single- and full-aperture-pair ion-optics models with each other and with experimental data. This study is the first to analyze the full-aperture-pair ion optics of an ion thruster and to clarify the range of applications and limitations of each ion-optics model.

\section{NUMERICAL MODEL}

\section{A. Configuration}

Simulations were performed on the MIPS ion optics. ${ }^{4,5}$ A cross-sectional view of the MIPS is shown in Fig. 3(a), in which plasma is generated in the discharge chamber by ECR heating using the ring-shaped permanent magnets and microwave antenna. Ions generated by the ECR heating enter the two-grid ion optics for acceleration.

The ECR heating process in MIPS was elucidated by Takao et al. using PIC-MCC simulations, and the ion current density and its velocity (average ion velocity of individual macro-particles) can be predicted with reasonable accuracy. ${ }^{6}$ Therefore, the whole region is divided into two (PIC-MCC and ion-optics simulation regions), and the simulation only focuses on the acceleration of ions using the information given by PIC-MCC calculations.
The simulation domain of the ion-optics of the MIPS aperture array is shown in Fig. 3(b). The MIPS aperture array consists of 211 apertures, and the six-fold hexagonal symmetry of the array allows the reduction of the simulation domain to $1 / 12$ th (24 apertures) of the whole aperture array. Each aperture is numbered from 1 to 24 to identify its location. The diameters of the screen and accel grid apertures are $0.8 \mathrm{~mm}$ and $0.4 \mathrm{~mm}$, respectively, and the distance between adjacent aperture centers is $1 \mathrm{~mm}$. The thicknesses of the screen and accel grid are $0.2 \mathrm{~mm}$ and $0.4 \mathrm{~mm}$, respectively. The distance between the screen and accel grids is $0.35 \mathrm{~mm}$.

\section{B. Assumption and modeling}

Ion-optics simulations were performed using the codes developed for the JAXA Ion Engine Development Initiatives (JIEDI) tool. ${ }^{17}$ The details of the JIEDI tool and its application to grid erosion analysis have been reported previously. ${ }^{17,18}$ The JIEDI tool is a set of Fortran90 codes: a mesh generation code, a neutral particle code, and an ion-optics code.

Figure 4 depicts a portion of the multi-aperture system of the MIPS ion optics. The neutral particle code tracks the flow of unionized propellant atoms. They are injected from the upstream boundary and exit through the grids in free molecular flow with the temperature of the grid surface. The injected flux of neutral atoms is uniform in the $x y$ direction, and the flux was determined from the beamlet current and propellant utilization efficiency.

The ion-optics code tracks an ion beamlet, which was developed based on the following general assumptions: (i) singly charged xenon ions $\mathrm{Xe}^{+}$and doubly charged xenon ions $\mathrm{Xe}^{2+}$ are considered, (ii) electrons are treated as a fluid based on the Boltzmann relationship, and (iii) the reactions between ions and neutral particles are charge-exchange and elastic collisions. A flowchart of the ion-optics code is shown in Fig. 5.

In the ion-optics code, the electrostatic potential of each nodal point is calculated using Poisson's equation

$$
\nabla^{2} \phi=-\frac{\rho_{i}-\rho_{e}}{\varepsilon} .
$$

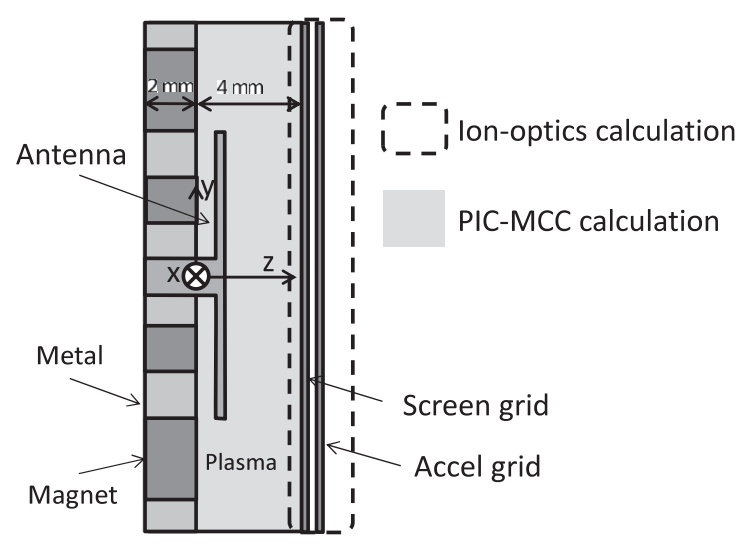

(a)

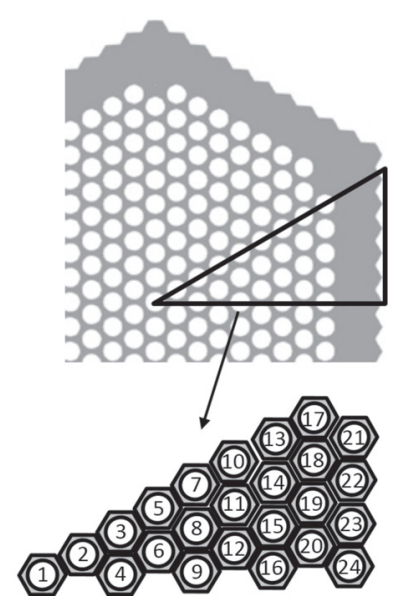

FIG. 3. Simulation domain for ion optics. (a) Cross-sectional view of the discharge chamber and grid pair and (b) front view of the 211-aperture-pair two-grid ion-optics set and the simulation domain $\left(30^{\circ} \times 60^{\circ}\right.$ right-triangle cross section) with a six-fold hexagonal symmetry. The apertures are numbered from 1 to 24 . 


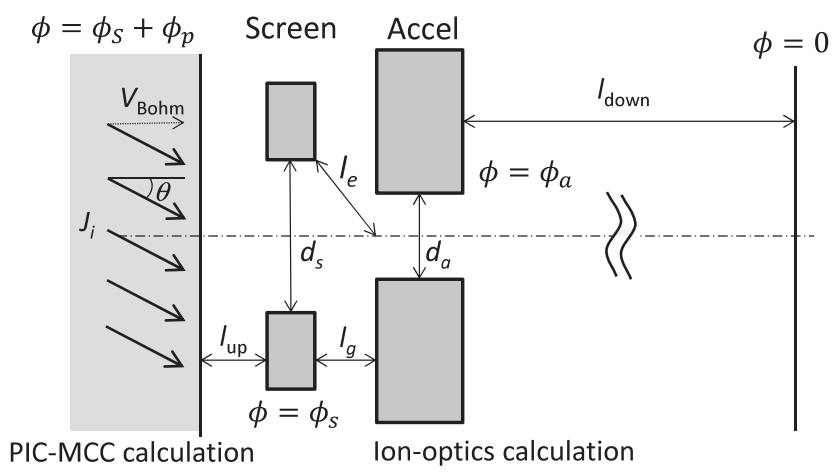

FIG. 4. Simulation domain of ion-optics calculation. Information at the upstream boundary is given by PIC-MCC calculations.

The charge neutrality condition in the discharge chamber plasma and the downstream neutralization plasma is satisfied using the Boltzmann relationship as follows:

$$
\rho_{e}=\rho_{e 0} e^{\frac{e\left(\phi-\phi_{p}\right)}{k_{B} T_{e}}} .
$$

The electron density in the discharge chamber is calculated using $\rho_{e 0}=m_{X e} J_{u p} /\left(e A_{u p} V_{B o h m}\right)$, and the electron density in the downstream neutralization plasma is given by $\rho_{e 0}$ $=m_{X e} J_{b} /\left(e A_{\text {down }} V_{\infty}\right)$. Equation (1) is coupled with Eq. (2) and solved by the finite element method. The simultaneous equations associated with all the nodal points are solved by the incomplete Cholesky conjugate gradient (ICCG) method with algebraic multi-block (AMB) ordering. ${ }^{19}$ Ion and neutral motion is tracked by the 4th-order Runge-Kutta method. A flux-tube concept is employed in which an ion is treated as a flux and its charge is distributed in the pairs of nodal points adjacent to its position.

Prior to ion-flux tracking, PIC-MCC simulation is performed to obtain the distribution of ion current density and ion velocity angles at the inlet boundary of the ion-optics simulation domain. The ion velocity angles were obtained by averaging the slopes of the velocity of individual macroparticles in the PIC-MCC simulation. These data were supplied on $0.1 \mathrm{~mm} \times 0.1 \mathrm{~mm}$ grid points on the $x y$ plane, and the values between them were linearly interpolated. The normalized ion current density and ion velocity angle (measured from the axial direction) averaged for each aperture are shown in Figs. 6(a) and 6(b). As shown in the figures, the ion current density has a ring-shaped, inhomogeneous distribution. The absolute value of ion current density is determined so that the ion beam currents passing through the ion-acceleration system (referred to as the beamlet current hereafter) equal a specified value. At the inlet boundary, the axial velocity of ions is set to be the Bohm velocity of the discharge chamber plasma with the incidence angles given by the PIC-MCC simulations.

In contrast, the distributions of the plasma potential and electron temperature obtained by the PIC-MCC simulations are uniform and flat, as shown in Fig. 6(c). Thus, the plasma potential and electron temperature in the discharge chamber were averaged and set to $26 \mathrm{~V}$ and $12 \mathrm{eV}$, respectively, to meet the MIPS operating conditions. The electron temperature in the downstream neutralization plasma was also set to $12 \mathrm{eV}$ because the MIPS neutralizer is driven by the same ECR

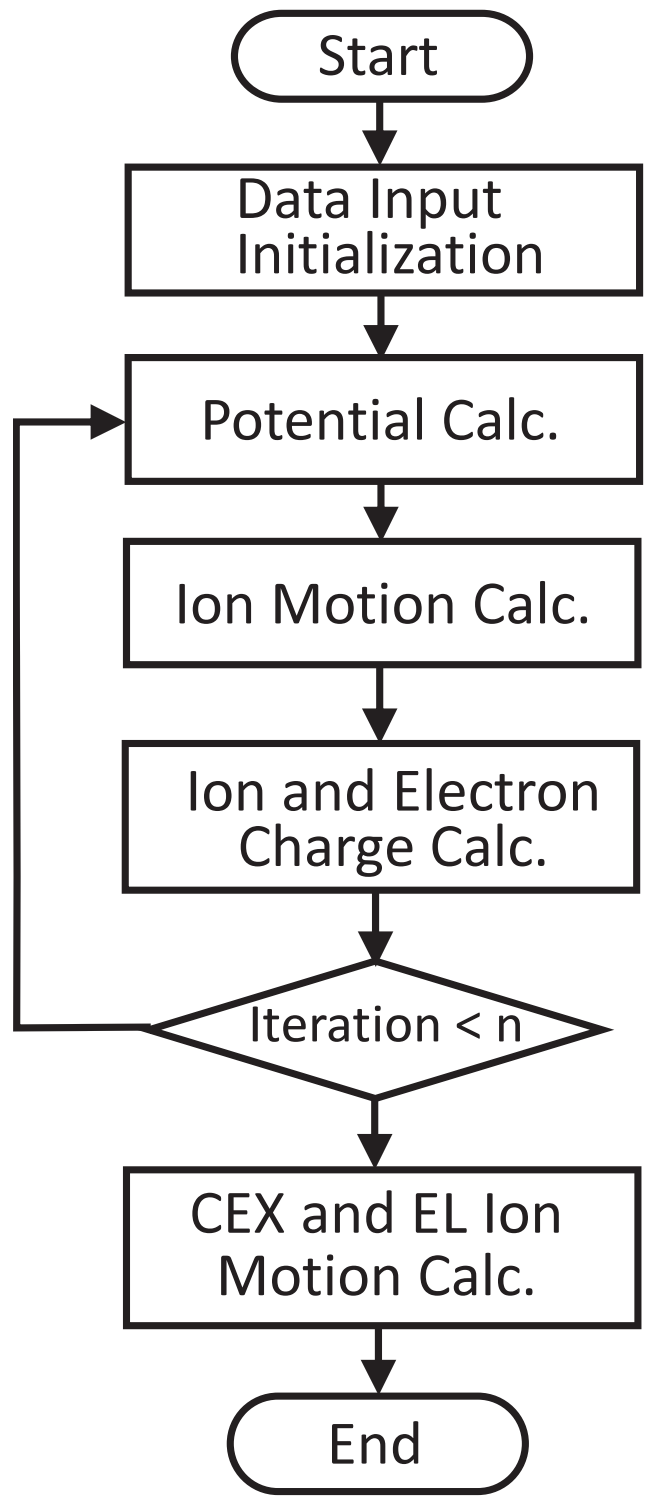

FIG. 5. Flowchart of the ion-optics calculation.

discharge. The interface between the PIC-MCC and ion-optics simulation regions is located $0.25 \mathrm{~mm}$ upstream of the screen grid surface. The distance to the downstream boundary from the accel grid is set to twice the distance to the potential hill, which is given by the one-dimensional $\operatorname{model}^{20}$ as

$$
l_{n}=\sqrt{\frac{1+3 R^{0.5}-4 R^{1.5}}{J_{b} / J_{s c l}}} l_{e} .
$$

As shown in Fig. 7, the ion-beam profile further downstream of this downstream boundary is calculated by summing all the beamlet currents after extrapolating their trajectories with straight ${ }_{L}$ lines. Each beamlet current is attenuated by a factor of $e^{-\int_{0}^{L} \sigma_{c e x} n_{n} d l}$ owing to the charge-exchange collisions with the residual neutral atoms in the vacuum chamber.

\section{Ion-optics models}

In this study, three ion-optics models were compared: (a) the averaged-beamlet-current single-aperture-pair ion-optics 
(a)

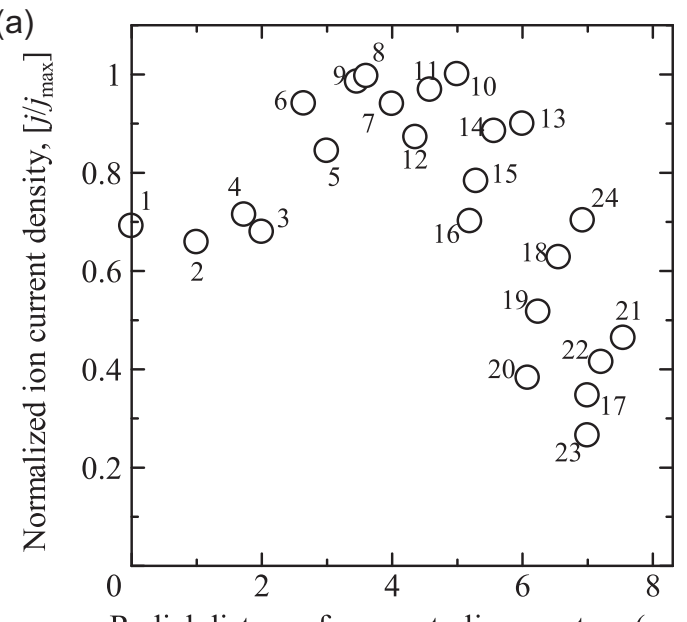

Radial distance from centerline aperture $(\mathrm{mm})$

(b)
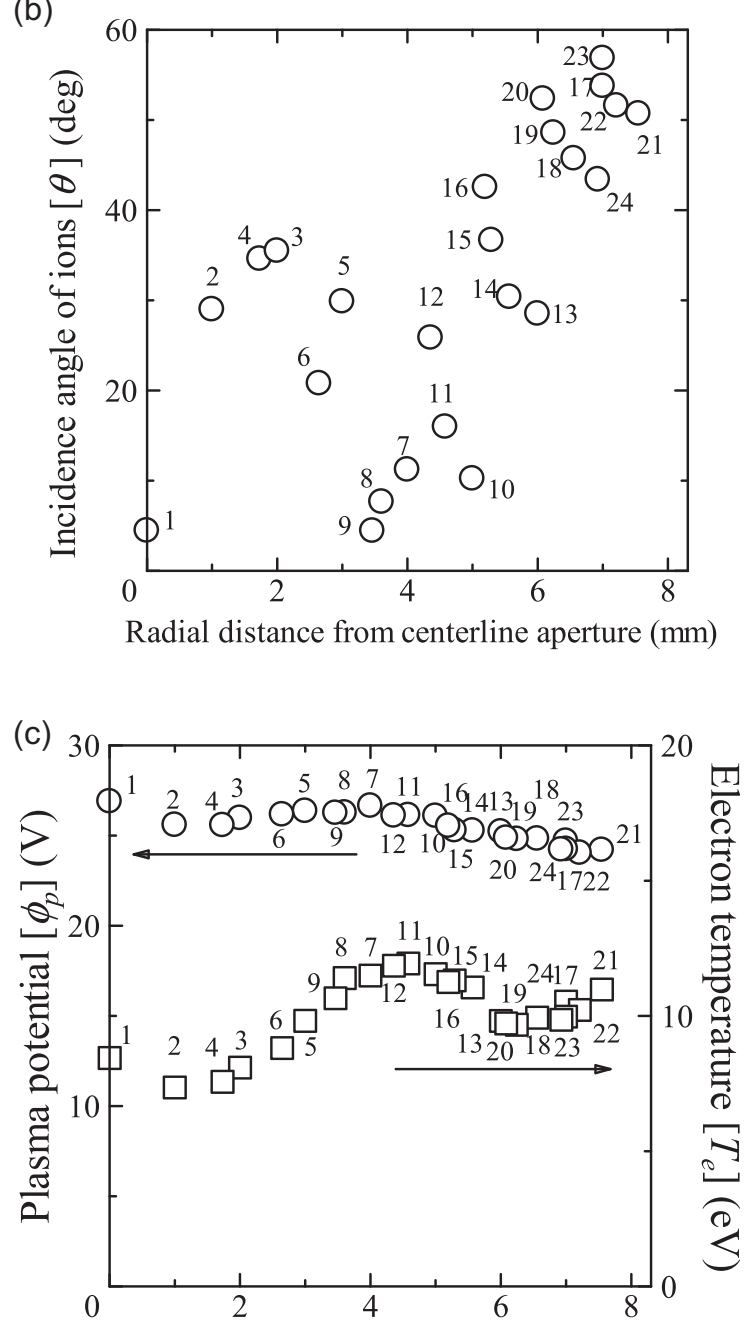

Radial distance from centerline aperture ( $\mathrm{mm}$ )

FIG. 6. (a) Distribution of ion current density (normalized by the maximum value) and (b) ion velocity angle for each aperture obtained by the PICMCC calculation.

model (referred to as the SA1 ion-optics model hereafter), (b) the 24 sets of single-aperture-pair ion-optics models (referred to as the SA24 ion-optics model hereafter), and (c) the fullaperture-pair ion-optics model (referred to as the FA ionoptics model hereafter).

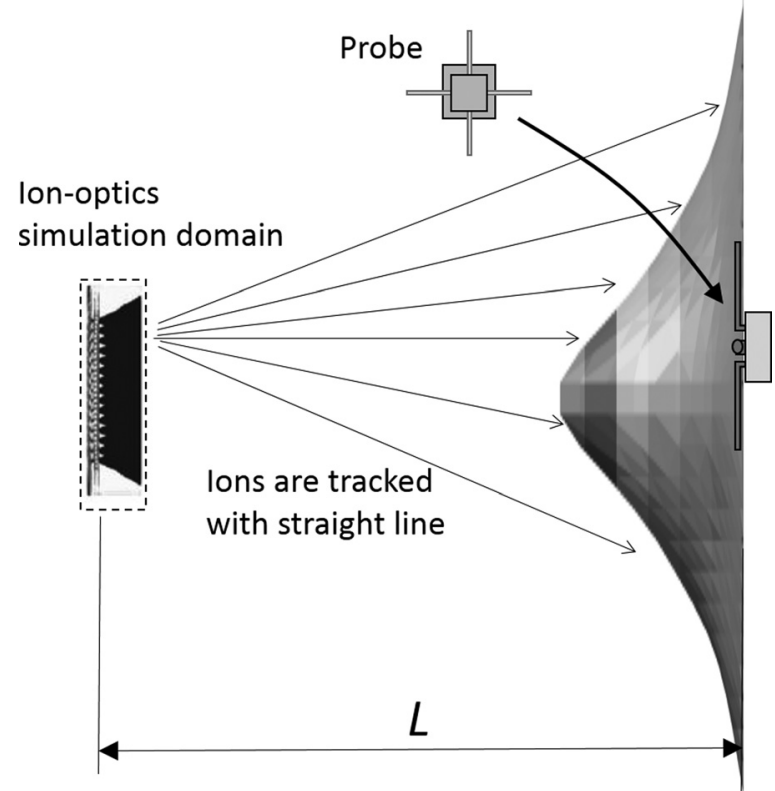

FIG. 7. Modeling of downstream ion beam current measurement.

Single-aperture-pair ion-optics models (referred to as SA ion-optics models hereafter) were used in the majority of previous ion-optics simulations and the SA1 ion-optics model is the simplest among them because its beamlet current is the average of beamlet currents of all apertures. The ion current density within each aperture in the SA24 ionoptics model is set constant, and its value was determined using the normalized ion current density distribution given by the PIC-MCC calculations so that the total beamlet current has the specified value. The beamlet current in the FA ion-optics model is determined in the same manner as that of the SA24 ion-optics model, except that the ion current density varies within each aperture, as calculated by the interpolation of the data obtained by the PIC-MCC calculations.

In all the ion-optics models, total beamlet currents are set to be equal. The ion incidence angles obtained by the PIC-MCC calculations [also shown in Fig. 6(b)] were used as the incidence angle of ions for the FA ion-optics model after interpolation to provide continuous distributions within an aperture. The incidence angles of ions were set to be $0^{\circ}$ in the SA1 and SA24 ion-optics models because they are hexagonally symmetric models.

On comparing the degree of approximation and the computational load of three ion-optics models, the SA1 ionoptics model is the most approximated model, but it is computationally least intensive. The SA24 ion-optics model is less approximated than the SA1 ion-optics model, but its computational cost is 24 times that of the SA1 ion-optics model. The FA ion-optics model is the most accurate model, but it is computationally the most intensive model among the three ion-optics models.

\section{Simulation domain and FEM model}

The simulation domain in the SA ion-optics models (SA1 and SA24 ion-optics models) is a hexagonal prism, and symmetric boundary conditions were imposed at the surface 
Single-aperture-pair model

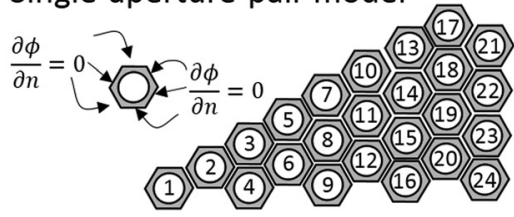

FEM mesh construction

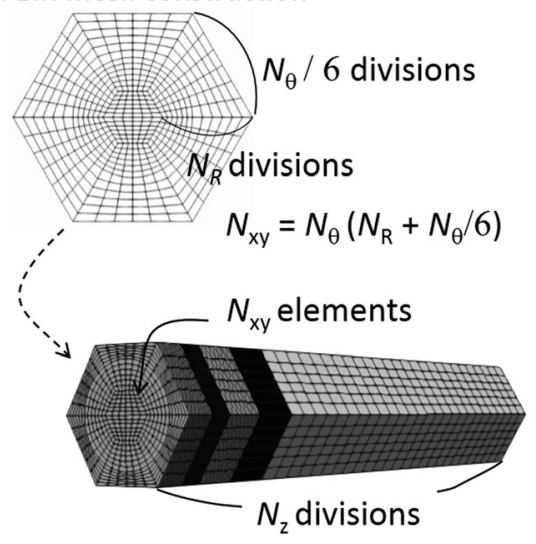

(a)
Full-aperture-pair model

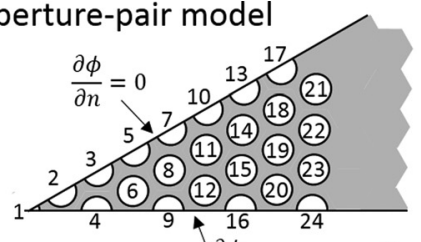

FEM mesh construction $\frac{\partial \phi}{\partial n}=0$

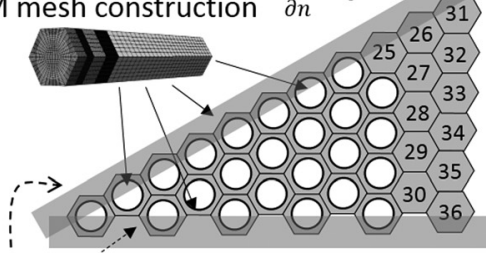

Removing edge elements

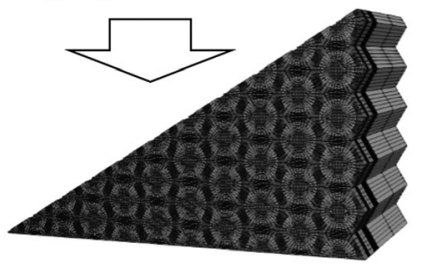

FIG. 8. (a) Single-aperture-pair ionoptics model and (b) full-aperture-pair ion-optics model. The middle and lower figures show the FEM model. of adjacent apertures (Fig. 8). In the FA ion-optics model, the simulation domain is constructed using the same hexagonal prism as for the SA ion-optics models and consists of 24 open-aperture hexagonal prisms (aperture Nos. 1-24) and 12 blind-aperture hexagonal prisms (aperture Nos. 25-36). No six-fold hexagonal symmetry was imposed between the apertures in the FA ion-optics model. The use of hexagonal prisms for the construction of the full-aperture array has the merit of good extensibility to grid sets of other numbers of apertures, such as 19- and 163-aperture grid sets. ${ }^{16,21}$

In all the ion-optics models, the simulation domain is discretized using 8-node hexahedral elements. The SA ionoptics domain is divided into $N_{\mathrm{xy}} \times N_{\mathrm{z}}$ elements, and the FA ion-optics domain is modelled using 36 SA ion-optics domains and by removing the elements in the edges, as shown in Fig. 8(b). The nodal distance and its accuracy in terms of the simulation results will be discussed by changing the mesh number in Sec. IIE.

\section{E. Mesh convergence}

Initial simulation runs were performed on the SA ionoptics model using the parameters listed in the left column of Table I for the MIPS grid set. The axial node spacing in the plasma region was set to be $70 \%-100 \%$ of the Debye

TABLE I. Operating parameters for (a) accel impingement current and (b) beam profile measurements.

\begin{tabular}{lcc}
\hline & Impingement current measurement & Beam profile measurement \\
\hline$J_{\mathrm{b}}(\mathrm{mA})$ & 2.76 & 5.5 \\
$P_{\mathrm{b}}(\mathrm{Pa})$ & $8 \times 10^{-3}$ & $8 \times 10^{-3}$ \\
$T_{\mathrm{g}}(\mathrm{K})$ & 290 & 290 \\
$\gamma$ & 0.10 & 0.10 \\
$\eta_{\mathrm{u}}(\%)$ & 31 & 39 \\
\hline \hline
\end{tabular}

(b)

length of the local plasma. Other node spacings were determined in such a way that the change in the accel impingement current becomes reasonably small using Richardson extrapolation.

Figure 9 compares the accel impingement current as a function of the number of axial divisions $N_{\mathrm{z}}(=59,70,140)$ for different numbers of surface divisions $N_{\mathrm{xy}}(=350,504$, 686 ) at an accel grid voltage of $-500 \mathrm{~V}$. The effect of surface divisions was investigated by changing the radial and circumferential divisions of $N_{\mathrm{R}}$ and $N_{\theta}$, as shown in Fig. 9, and a reasonable convergence can be observed on increasing $N_{\mathrm{xy}}$ and $N_{\mathrm{z}}$. The plots in Fig. 9 have the trend of $J_{a}\left(N_{x y}, N_{z}\right)$ $\approx J_{a}\left(\infty, N_{z}\right)+c_{x y}\left(1 / N_{x y}\right)$ and $J_{a}\left(N_{x y}, N_{z}\right) \approx J_{a}\left(N_{x y}, \infty\right)$ $+c_{z}\left(1 / N_{z}^{2}\right)$, where $c_{\mathrm{xy}}$ and $c_{\mathrm{z}}$ are constants.

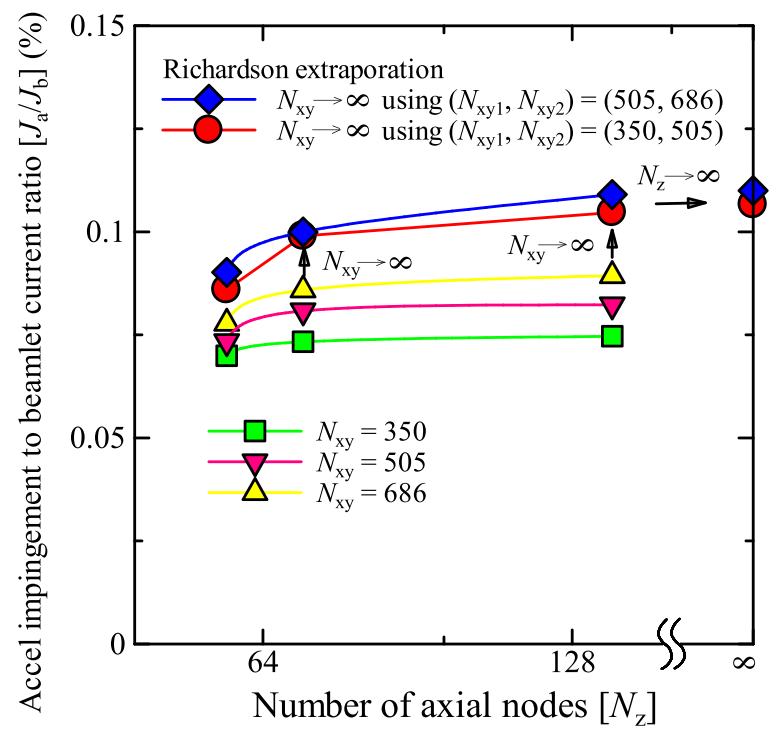

FIG. 9. Accel impingement current and the number of divisions. Richardson extrapolation using $\left(N_{\mathrm{xy}}, N_{\mathrm{z}}\right)=(505,70),(505,140),(686,70)$, and $(686$, $140)$ is employed to estimate the mesh-convergent solution. 
A simple method to obtain a mesh-convergent solution is to increase the number of divisions; however, increasing $N_{\text {xy }}$ and $N_{\mathrm{z}}$ leads to a dramatic increase of computational cost in the FA ion-optics model. Therefore, to reduce computation cost, plots of Richardson extrapolation are used to estimate the accel impingement current for $N_{\mathrm{xy}} \rightarrow \infty$, which is given by

$$
J_{a}\left(\infty, N_{z}\right)=J_{a}\left(N_{x y 2}, N_{z}\right)+\frac{J_{a}\left(N_{x y 2}, N_{z}\right)-J_{a}\left(N_{x y 1}, N_{z}\right)}{\left(\sqrt{N_{x y 1} / N_{x y 2}}\right)^{2}-1} .
$$

The top two lines in Fig. 9 show the curves obtained by Richardson extrapolation using $\left(N_{\mathrm{xy} 1}, N_{\mathrm{xy} 2}\right)=(350,686)$ and (504, 686), and they are very close to each other. Using the same strategy, a mesh-convergent solution for the $z$-direction $\left(N_{\mathrm{z}} \rightarrow \infty\right)$ is obtained using Richardson extrapolation as follows:

$$
J_{a}(\infty, \infty)=J_{a}\left(\infty, N_{z 2}\right)+\frac{J_{a}\left(\infty, N_{z 2}\right)-J_{a}\left(\infty, N_{z 1}\right)}{\left(N_{z 1} / N_{z 2}\right)^{2}-1} .
$$

In this case, the accel impingement current for $N_{\mathrm{xy}} \rightarrow \infty$ and $N_{\mathrm{z}} \rightarrow \infty$ is $0.11 \%$ using two points $\left(N_{\mathrm{z} 1}=70\right.$ and $\left.N_{\mathrm{z} 2}=140\right)$ on the line obtained by the Richardson extrapolation using $\left(N_{\mathrm{xy} 1}, N_{\mathrm{xy} 2}\right)=(504,686)$. This division combination was selected because the change in $J_{a}(\infty, \infty)$ becomes less than $5 \%$ on doubling $N_{\mathrm{xy}}$ and $N_{\mathrm{z}}$. The same strategy and the same number of divisions in the $36 \mathrm{SA}$ ion-optics domains were used for the calculation in the FA ion-optics model, which helps estimate the mesh-convergent solution in a reasonable time and computational cost.

\section{EXPERIMENTS}

For comparison, the accel impingement current as a function of accel grid voltage and the ion-current profile downstream of the thruster are used, and they were measured using the MIPS grid set. In the experiments, the screen grid voltage was set to be $1500 \mathrm{~V}$, and the accel grid voltage for the ion-beam profile measurement was $-350 \mathrm{~V}$. In the accel impingement current measurements, the accel grid voltage was changed from $-100 \mathrm{~V}$ to $-500 \mathrm{~V}$. Other operating parameters for the accel impingement current and ion-current profile measurements are listed in Table I. The doubly charged ion fraction was estimated from the measurements conducted on a similar microwave discharge ion thruster. ${ }^{22}$

Measurements were conducted in a vacuum chamber evacuated by a turbo-molecular pump. The pre-test pressure in the chamber was $3 \times 10^{-3} \mathrm{~Pa}$ and the pressure was maintained at $8 \times 10^{-3} \mathrm{~Pa}$ during the measurements. The accumulated operating time of the grid set before the measurements was approximately $130 \mathrm{~h}$. The ion-current profiles were obtained $300 \mathrm{~mm}$ downstream of the thruster using a probe with an effective area of $32 \mathrm{~mm}^{2}$. The ion current due to residual plasma in the discharge chamber is estimated to be approximately $5 \%$ of the peak ion current measured in the experiments.

\section{RESULTS AND DISCUSSION}

\section{A. Accel impingement current}

Figure 10 shows a comparison of accel impingement current as a function of the magnitude of accel grid voltage. Simulations were performed using the parameters listed in the left column of Table I. As shown in Fig. 10, the accel impingement current measured in the experiment increases with the magnitude of accel grid voltage. This result is consistent with crossover impingement observed at higher net accelerating voltages in an ion thruster. In Fig. 10, all the curves obtained from the numerical results appear to follow the same general trend in which the accel impingement current increases with the magnitude of accel grid voltage. It is clear from a comparison of the three ion-optics models that the FA ion-optics model yields results that quantitatively agree best with the experimental data. The slight overprediction against the experimental data can be explained by the increase of diameter of the accel grid aperture by sputter erosion during experiments, which decreased the accel impingement current.

As shown in Fig. 10, the SA ion-optics models gave reasonably good results at a higher magnitude of accel grid voltage, but they tend to underestimate the results of the FA ionoptics model. The difference between the two SA ion-optics models is very small, which means that the SA1 ion-optics model is convenient for a rough and quick estimation of accel impingement current because it can yield results similar to those obtained using the SA24 ion-optics model with only $1 / 24$ th of the computational cost.

To explain the differences among the ion-optics models, the accel impingement current was divided into two components: that due to the direct impingement of beamlet ions and that due to the impingement of ions produced by chargeexchange (CE) and elastic (EL) collisions between the beamlet ions and neutral particles. Figure 11(a) shows a comparison of the direct impingement current. The direct

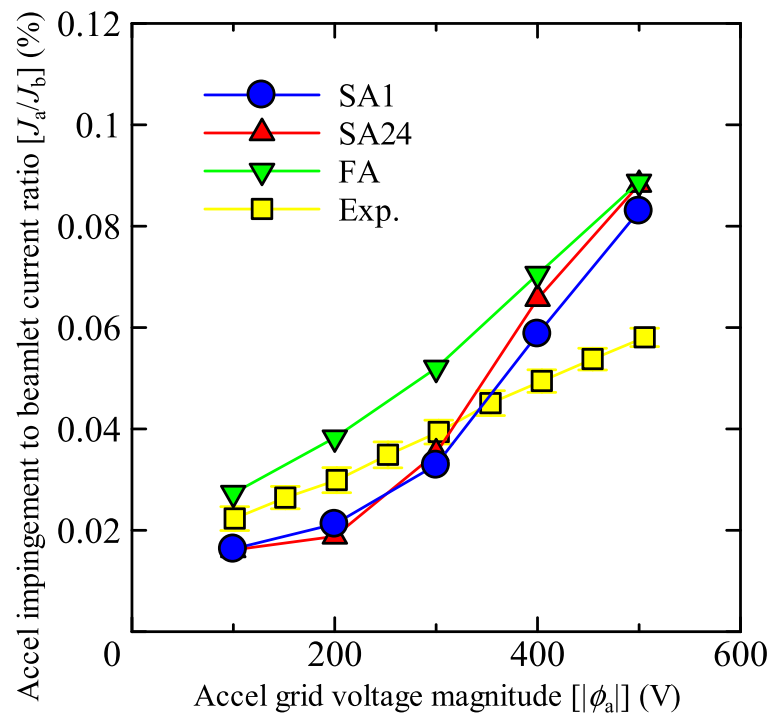

FIG. 10. Comparison of the ratio of accel impingement current to beamlet current among the SA1 ion-optics model, SA24 ion-optics model, FA ionoptics model, and experiment. 
(a)

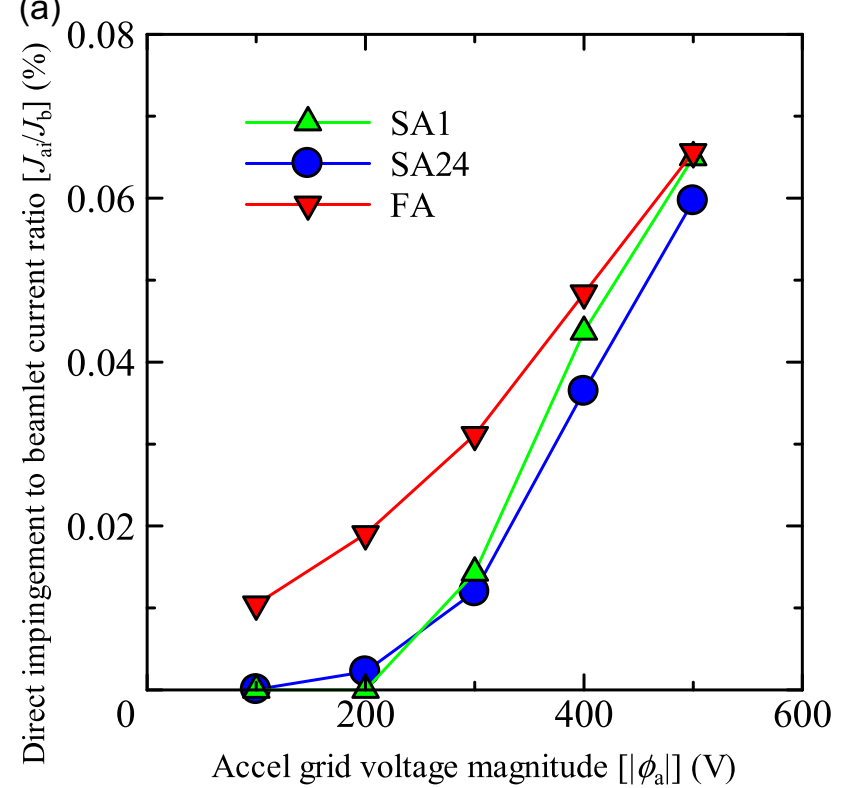

(b)

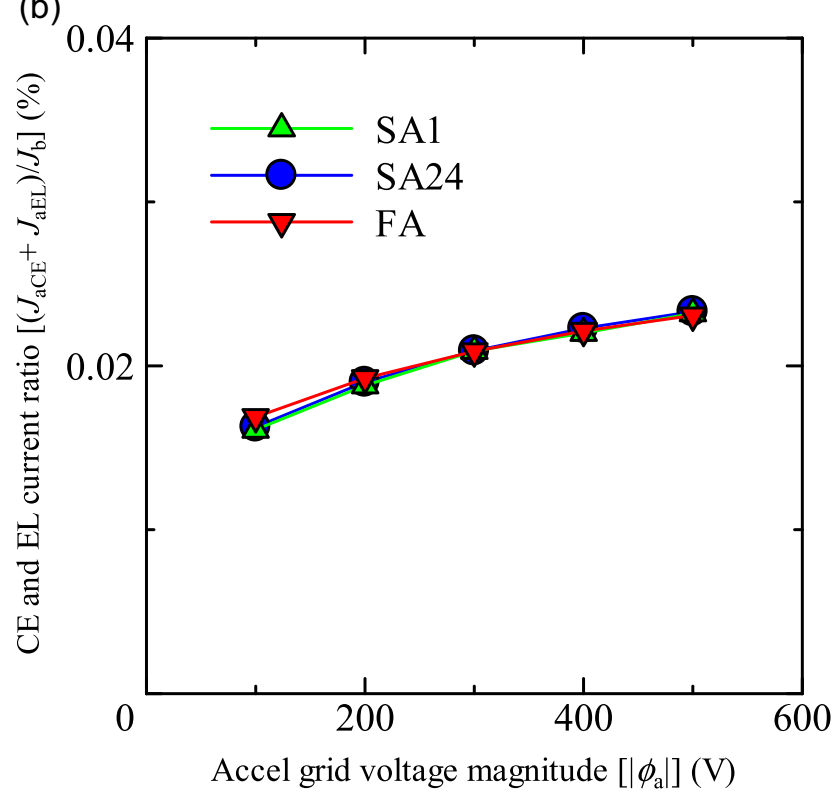

FIG. 11. Comparison of (a) the direct impingement current and (b) the ratio of charge-exchange and elastic-collision ion current to the beamlet current among the SA1, SA24, and FA ion-optics models.

impingement current predicted by the FA ion-optics model is higher than those predicted by the SA ion-optics models. In contrast, the calculated CE and EL ion currents shown in Fig. 11(b) were very close to each other. These results suggest that the difference of direct impingement current is the source of the difference of accel impingement current.

In order to investigate the difference of accel impingement current between apertures, the direct impingement currents and the CE and EL ion currents for $\phi_{\mathrm{a}}=-300 \mathrm{~V}$ and $-500 \mathrm{~V}$ were compared. To elucidate the angle dependence of incident ions in the FA ion-optics model, additional calculations were performed by setting the incidence angle of ions to 0 (referred to as the FA $(\theta=0)$ ion-optics model). As shown in Figs. 12(a) and 13(a), the direct impingement current in the FA ion-optics model is higher in the center and

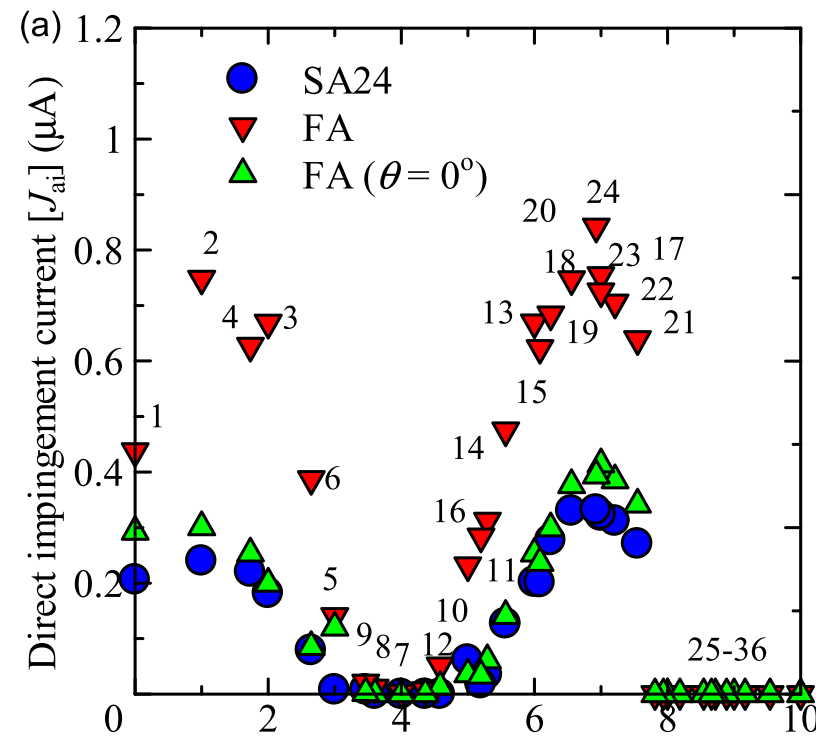

Radial distance from centerline aperture (mm)

(b)

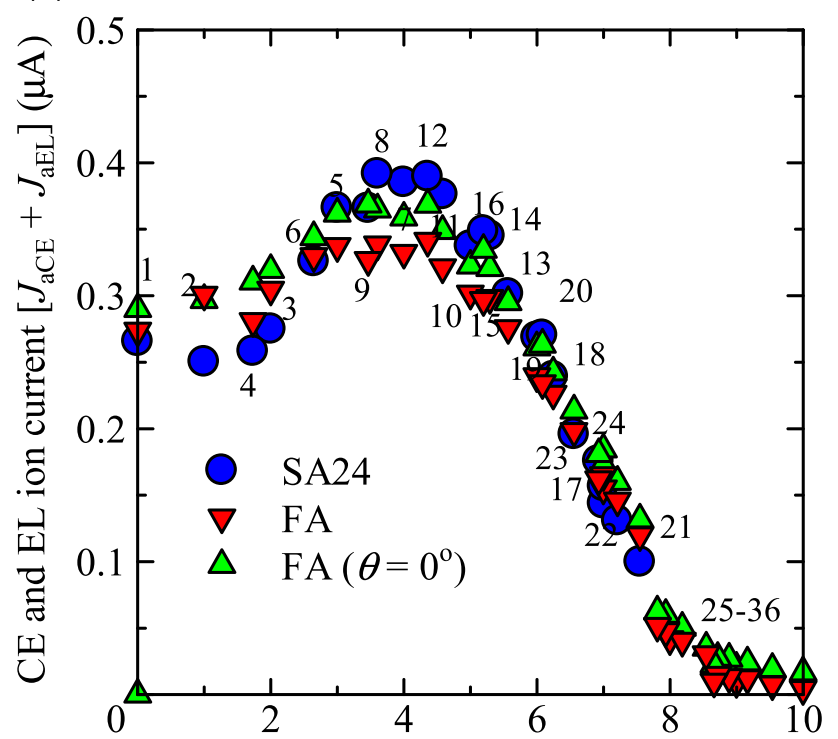

Radial distance from centerline aperture ( $\mathrm{mm})$

FIG. 12. Comparison of (a) direct impingement current and (b) chargeexchange and elastic-collision ion current for each aperture between the SA24 and FA ion-optics models. The accel grid voltage is $-300 \mathrm{~V}$.

periphery regions, but the FA $\left(\theta=0^{\circ}\right)$ ion-optics model yielded almost the same results as those of the SA24 ionoptics model. This result shows that the oblique incidence angle of ions in the center and periphery region of MIPS is the source of the large direct impingement current.

In contrast, the $\mathrm{CE}$ and EL ion currents shown in Figs. 12(b) and 13(b) are similar, and the FA $\left(\theta=0^{\circ}\right)$ ion-optics model yields results close to those of the SA24 and FA ionoptics models. This result indicates that the incidence angle of ions has little impact on the CE and EL ion currents because the initial velocity of $\mathrm{CE}$ ions depends only on the velocity of neutral atoms. It should be noted that the FA ionoptics model yielded slightly lower peak currents than the SA24 ion-optics model in Figs. 12(b) and 13(b). The main reason for this difference is the current flowing outward to 
(a)

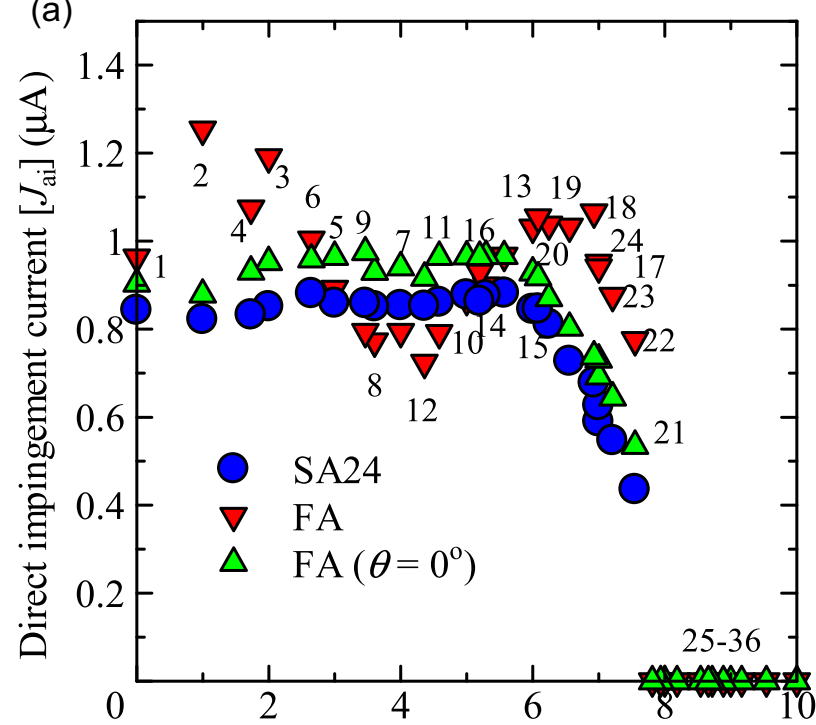

Radial distance from centerline aperture ( $\mathrm{mm})$

(b)

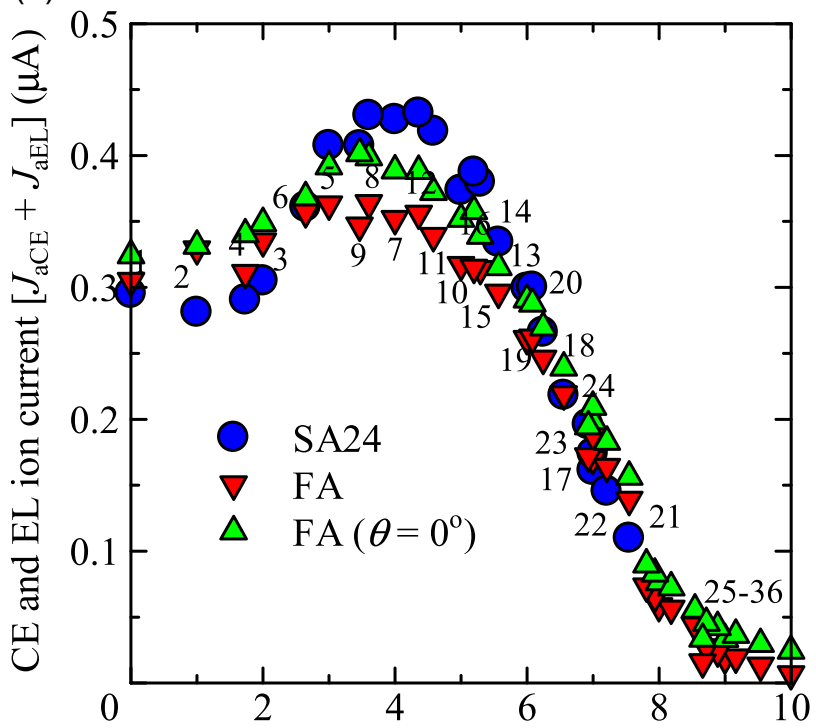

Radial distance from centerline aperture (mm)

FIG. 13. Comparison of (a) direct impingement current and (b) chargeexchange and elastic-collision ion current for each aperture between the SA24 and FA ion-optics models. The accel grid voltage is $-500 \mathrm{~V}$.

the periphery region of the grid (blind aperture Nos. 25-36), which is only included in the FA ion-optics model.

From these results, even though the predicted total accel impingement currents appear similar, the distribution of accel impingent current for each aperture is different among the ion-optics models. The FA ion-optics model yielded the most accurate results because only this model can deal with the oblique incidence angles of ions in the very inhomogeneous MIPS plasma source and blind aperture region. Therefore, the FA ion-optics simulation is better suited to identify the bottleneck point in the MIPS grid set and helps to design and optimize its ion optics. It should be mentioned that the majority of ion-optics simulations conducted so far yielded reasonable results with experiments using the SA ion-optics model because the incidence angles of ions in the
MIPS plasma source are very oblique compared to those in other plasma sources. In addition, its effect could be quantitatively clarified with PIC-MCC simulations, which provided abundant quantitative plasma data that had not been obtained in previous studies.

\section{B. Beam-current profile}

Simulations were performed to compare the beamcurrent profiles for the operating conditions listed in the right column of Table I. ${ }^{23}$ Figure 14 shows a comparison of beamcurrent profiles $300 \mathrm{~mm}$ downstream of the accel grid, in which the beamlet current is attenuated by approximately $20 \%$ before reaching the probe by the charge-exchange collisions between the ions and ambient neutral particles in the vacuum chamber. In the calculation, the beam-current profile was simulated by multiplying the ion current density with the probe area of $32 \mathrm{~mm}^{2}$. The ion current due to residual plasma was ignored since its value was measured to be approximately $5 \%$ of the peak current in the experiment.

As shown in the figure, the calculated beam-current profiles are similar in shape. The integrated beam current of each ion-optics model yielded the same value of $840 \mu \mathrm{A}$, and it was $770 \mu \mathrm{A}$ in the experiment. Cross-sectional plots of ion-beam profile on the $y z$-plane are shown in Fig. 15. Although the integrated beam currents were identical, peak currents are different among the SA24, SA1, and FA ionoptics models; the SA ion-optics models yield sharp highpeak profiles, whereas the FA ion-optics model yields a rather flat and wide profile.

Because the ion-beam profile measurement has some uncertainties, a direct comparison of beam current between the ion-optics models and the experiment is meaningless. Therefore, the beam-divergence angle is compared, which is defined by the cone angle containing $95 \%$ of the total beamlet current. The beam-divergence angle calculated from the experimental data was $25.3^{\circ}$, whereas the calculated beamdivergence angle is approximately $23^{\circ}$ in the SA1 and SA24 ion-optics models and $24.5^{\circ}$ in the FA ion-optics model. The angle obtained by the FA ion-optics model is the widest among the three ion-optics models and shows the best agreement with experimental data. Since the information of ionbeam profile and beam-divergence angle is vital for the evaluation of spacecraft-plasma interactions and the underestimation of the envelope angle of the ion beam is not preferred, the FA ion-optics model is most suited for the evaluation of ion-beam profiles.

\section{CONCLUSIONS}

A simulation of the 211-aperture-pair two-grid ionoptics for the miniature ion propulsion system (MIPS) was performed using the ion-current distribution and ion velocity angles given by PIC-MCC simulation. Since the MIPS plasma source is too inhomogeneous to introduce mirror or translational boundary conditions that reduce simulation regions, all the aperture pairs of the MIPS grid set were analyzed self-consistently.

To demonstrate the effectiveness of full-aperture-pair ion-optics simulations, the accel impingement current 

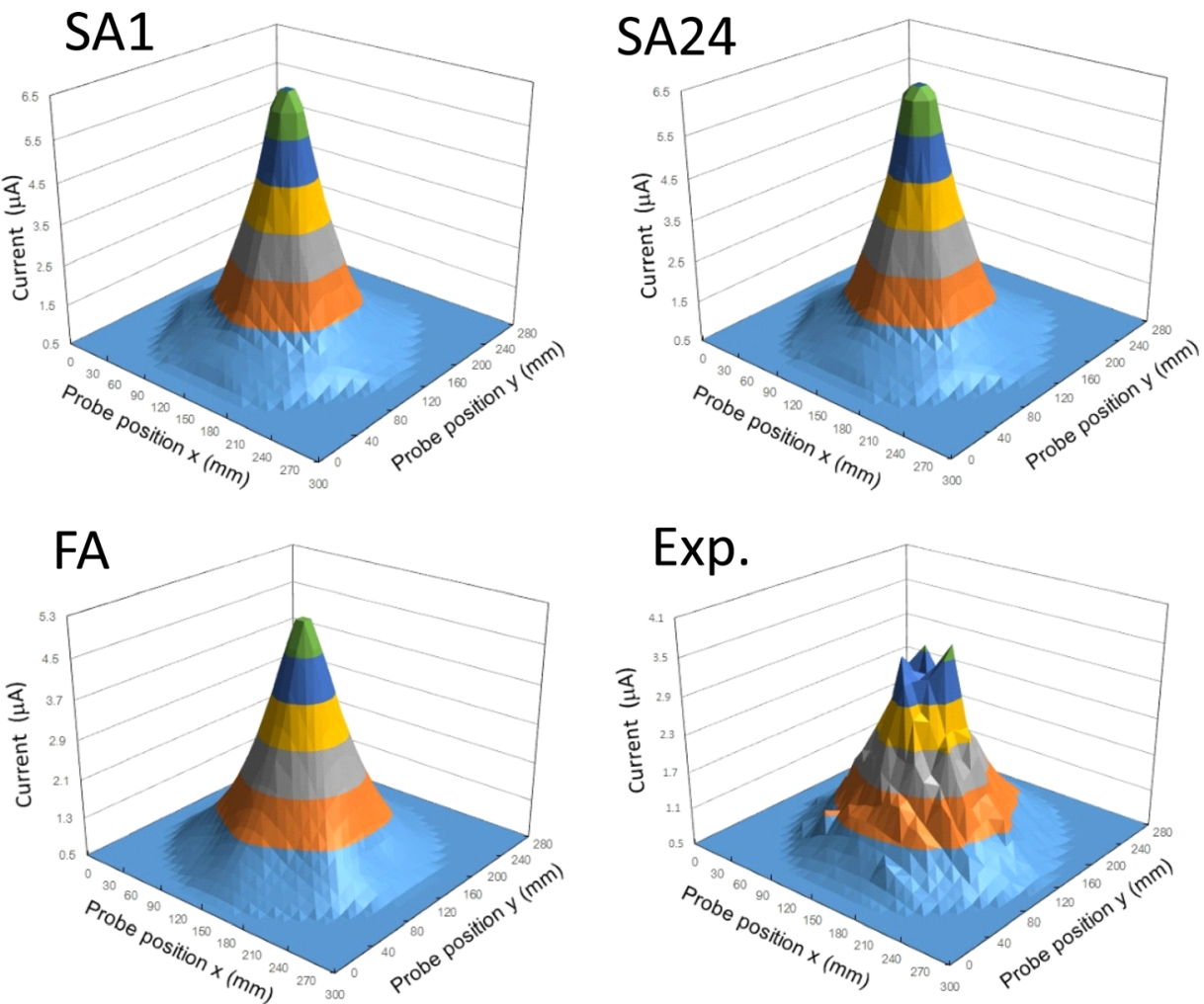

FIG. 14. Beam-current profile $300 \mathrm{~mm}$ downstream of the thruster for (a) the SA1 ion-optics model, (b) the SA24 ion-optics model, (c) the FA ion-optics model, and (d) experiment. obtained using the simulations was compared to that obtained experimentally and those obtained by the singleaperture-pair ion-optics models used in the majority of previous ion-optics simulations. The accel impingement current calculated as a function of accel grid voltage shows a trend similar to that obtained experimentally. The agreement in the full-aperture-pair ion-optics model is satisfactory, whereas the single-aperture-pair ion-optics models provide conservative predictions. The difference of direct impingement current was found to be the source of this difference,

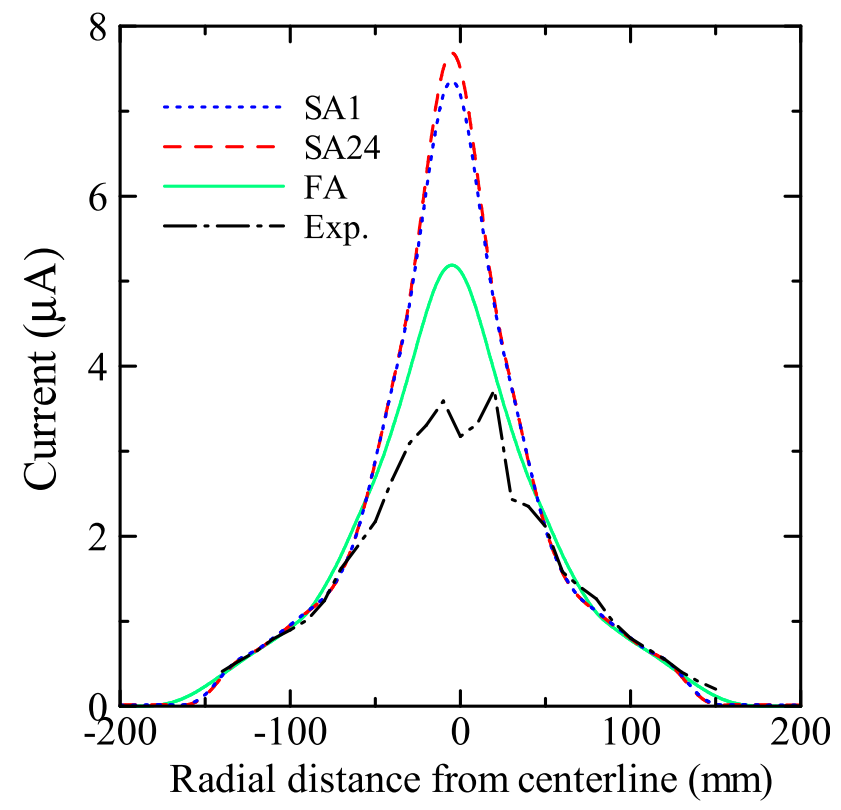

FIG. 15. Beam-current profiles on the yz-plane for the SA1, SA24, and FA ion-optics models and the experiment. and the direct impingement was greatly affected by the incidence angle of ions at the inlet boundary. In contrast, little differences were found between the ion currents produced by charge-exchange and elastic collisions. A comparison of the beam profile $300 \mathrm{~mm}$ downstream of the thruster between the experiment and the simulations showed reasonable agreement in the case of the full-aperture-pair ion-optics model.

Comparative studies of ion-optics models showed that the full-aperture-pair ion-optics model yielded the most accurate results for the MIPS grid set because the MIPS plasma source is highly inhomogeneous, but the singleaperture ion-optics models can yield reasonable results for typical ion thrusters. Therefore, it is concluded that full-aperture-pair ion-optics simulations provide a clear understanding of the ion impingement phenomena of the MIPS grid set and help to design ion thrusters with highly inhomogeneous plasma sources effectively and accurately by a numerical approach.

\section{ACKNOWLEDGMENTS}

This work was financially supported in part by a Grantin-Aid for Scientific Research (S), 16H06370, from the Japan Society for the Promotion of Science.

${ }^{1}$ C. Casaregola, "Electric propulsion for station keeping and electric orbit raising on Eutelsat platforms," in 34th International Electric Propulsion Conference (Kobe, Japan, 2015), Paper No. IEPC-2015-97.

${ }^{2}$ H. Kuninaka, K. Nishiyama, I. Funaki, T. Yamada, Y. Shimizu, and J. Kawaguchi, J. Propul. Power 23, 544 (2007).

${ }^{3}$ C. E. Garner, M. D. Rayman, and J. Brophy, "In-flight operation of the Dawn ion propulsion system-arrival at Ceres," in 34th International Electric Propulsion Conference (Kobe, Japan, 2015), Paper No. IEPC2015-88. 
${ }^{4}$ H. Koizumi, K. Komurasaki, J. Aoyama, and K. Yamaguchi, Trans. Jpn. Soc. Aeronaut. Space Sci., Aerosp. Technol. Jpn. 12, Tb_19 (2014).

${ }^{5}$ H. Koizumi, H. Kawahara, K. Yaginuma, J. Asakawa, Y. Nakagawa, Y. Nakamura, S. Kojima, T. Matsuguma, R. Funase, J. Nakatsuka, and K. Komurasaki, Trans. Jpn. Soc. Aeronaut. Space Sci., Aerosp. Technol. Jpn. 14, Pb_13 (2016).

${ }^{6}$ Y. Takao, H. Koizumi, K. Komurasaki, K. Eriguchi, and K. Ono, Plasma Sources Sci. Technol. 23, 064004 (2014).

${ }^{7}$ Y. Arakawa and K. Ishihara, "A numerical code for cusped ion thrusters," in 22nd International Electric Propulsion Conference (Viareggio, Italy, 1991), Paper No. IEPC-91-118.

${ }^{8}$ Y. Hayakawa, J. Propul. Power 8, 110 (1992).

${ }^{9}$ Y. Okawa, H. Takegahara, and T. Tachibana, "Numerical analysis of ion beam extraction phenomena in an ion thruster," in 27th International Electric Propulsion Conference (Pasadena, USA, 2001), Paper No. IEPC-01-97.

${ }^{10}$ Y. Nakayama and P. J. Wilbur, J. Propul. Power 19, 607 (2003).

${ }^{11}$ C. C. Farnell and J. D. Williams, J. Propul. Power 26, 125 (2010).

${ }^{12}$ A. Shagayda, V. Nikitin, and D. Tomilin, Vacuum 123, 140 (2016).

${ }^{13}$ J. Wang, J. Polk, J. Brophy, and I. Katz, J. Propul. Power 19, 1192 (2003).

${ }^{14}$ R. Kafafy and J. Wang, "Whole subscale ion optics simulation: Direct impingement and electron backstreaming," AIAA Paper No. 2005-3691, 2005.
${ }^{15} \mathrm{C}$. Farnell, "Performance and lifetime simulation of ion thruster optics," Ph.D. dissertation (Department of Aeronautics and Astronautics, Colorado State University, Boston, 2007).

${ }^{16}$ P. J. Wilbur, J. Miller, C. Farnell, and V. K. Rawlin, "A study of high specific impulse ion thruster optics," in 27th International Electric Propulsion Conference (Pasadena, USA, 2001), IEPC-01-098.

${ }^{17}$ M. Nakano, Y. Kajimura, and I. Funaki, Trans. Jpn. Soc. Aeronaut. Space Sci., Aerosp. Technol. Jpn. 10, Pb_85 (2012).

${ }^{18}$ H. Watanabe, M. Nakano, Y. Kajimura, and I. Funaki, Trans. Jpn. Soc. Aeronaut. Space Sci., Aerosp. Technol. Jpn. 12, Pb_65 (2014).

${ }^{19}$ T. Iwashita and M. Shimasaki, IEEE Trans. Magn. 38, 429 (2002).

${ }^{20}$ R. Kaufman, "One-dimensional analysis of ion rockets," Report No. NASA TN D-261, 1960.

${ }^{21}$ H. Hayashi, H. Kuninaka, M. Usui, Y. Shimizu, S. Hosoda, H. Koizumi, and K. Nishiyama, "R\&D on 10,000 sec isp ion engine driven by microwave," AIAA Paper No. 2008-4810, 2008.

${ }^{22}$ S. Satori, H. Kuninaka, and M. Otaki, Aeronaut. Space Sci. Jpn. 46, 406 (1998).

${ }^{23}$ K. Yaginuma, H. Koizumi, H. Kawahara, J. Asakawa, Y. Nakagawa, T. Inagaki, Y. Kasagi, S. Ikari, and N. Ozaki, J. Jpn. Soc. Aeronaut. Space Sci. 64, 131 (2016) 